Design Growth-arrested AFs were incubated in serum-free medium with UII and some inhibitors of signal transduction pathways. Cell migration was determined by a transwell technique. The OPN mRNA expression and protein secretion induced by UII were evaluated by the reverse transcriptase PCR and ELISA method, respectively.

Results OPN antisense oligonucleotides inhibited UII-induced AFs migration significantly compared with UII $\left(10^{-8} \mathrm{~mol} / \mathrm{l}\right)$ group $(p<0.05)$. Moreover, UII promoted the OPN mRNA expression and protein secretion in a dose-dependent and time-dependent manner, with maximal effect at a concentration of $10^{-8} \mathrm{~mol} / \mathrm{l}$ at $3 \mathrm{~h}$ for mRNA expression, or at $24 \mathrm{~h}$ for protein secretion, respectively $(p<0.01)$. The UII receptor antagonist SB710411 $\left(10^{-6} \mathrm{~mol} / \mathrm{l}\right), \mathrm{Ca}^{2+}$ channel blocker nicardipine $\left(10^{-5} \mathrm{~mol} / \mathrm{l}\right)$, protein kinase $\mathrm{C}$ inhibitor H7 $\left(10^{-5} \mathrm{~mol} / \mathrm{l}\right)$, calcineurin inhibitor cyclosporine A $\left(10^{-5} \mathrm{~mol} / \mathrm{l}\right)$, Rho kinase inhibitor Y-27632 $\left(10^{-5} \mathrm{~mol} / \mathrm{l}\right)$ and mitogen activated protein kinase (MAPK) inhibitor PD98059 $\left(10^{-5} \mathrm{~mol} / \mathrm{l}\right)$ inhibited the UII effects significantly.

Conclusion This study indicated that UII may up-regulate OPN expression in AFs through the UII receptor, protein kinase C, MAPK, calcineurin, Rho kinase and $\mathrm{Ca} 2+$ signal transduction pathways, and OPN is involved in UII-induced AFs migration.

\section{e0160 TRANSIENT PREHYPERTENSIVE TREATMENT IN SPONTANEOUSLY HYPERTENSIVE RATS:A COMPARISON OF LOSARTAN AND AMLODIPINE REGARDING LONG-TERM BLOOD PRESSURE AND RENAL PROTECTIVE EFFECT}

doi:10.1136/hrt.2010.208967.160

Jinxiu Lin, Hong Tang, Liming Lin, Changsheng Xu. Department of Cardiology, The First Affiliated Hospital of Fujian Medical University, Fujian Hypertension Research Institute, Fuzhou, China

Aims To compare the effectiveness of transient prehypertensive treatment with losartan vs amlodipine in spontaneously hypertensive rats (SHR) on long-term blood pressure and cardiac protection Main methods SHR were prehypertensively (weeks 4-10 of age) treated with losartan (SHR-Los: $20 \mathrm{mg} / \mathrm{kg} /$ day), amlodipine (SHR-Aml: $10 \mathrm{mg} / \mathrm{kg} / \mathrm{day}$ ) or saline ( $\mathrm{n}=24$ each group). Rats were followed up until week 46. Systolic blood pressure (SBP) was measured by tail-cuff method. Cardiac parameters including Left ventricular (LV) mass index (LVMI), collagen volume fraction (CVF) and LV function were assessed by histomorphometry and echocardiography. Plasma and myocardium Angiotensin II (Ang II) and aldosterone (Aldo) were measured by radioimmunoassay. Cardiac angiotensin II type 1 and type 2 receptor (AT1R and AT2R) protein were determined by immunoblotting and brain natriuretic peptide (BNP) mRNA was semi-quantified by reverse transcription-PCR (RT-PCR).

Key findings The SBP in SHR-Los was reduced until age 46 weeks, but returned to untreated SHR levels in SHR-Aml from 30 weeks onwards. Compared to untreated SHR, the LVMI and CVF in SHRLos were markedly decreased until week 46, and the LV ejection fraction (LVEF) (SHR-Los vs SHR: $83.1 \pm 2.3 \%$ vs $79.5 \pm 1.9 \%$, $\mathrm{p}<0.05$ ) and cardiac BNP mRNA expression were improved, whereas comparable LVMI and elevated CVF were found in SHR$\mathrm{Aml}$, and the LVEF fell significantly below that of untreated SHR at week 46 (SHR-Aml vs SHR: $74.4 \pm 4.3 \%$ vs $79.5 \pm 1.9 \%, p<0.05$ ), with cardiac BNP mRNA expression increasing slightly. Compared to untreated SHR, the plasma and myocardium AngIIand Aldo levels in SHR-Los at week 46 were remarkably decreased (plasma AngII: $302 \pm 32$ vs $458 \pm 32 \mathrm{pg} / \mathrm{ml}$; plasma Aldo: $172 \pm 20$ vs $252 \pm 41 \mathrm{pg} / \mathrm{ml}$; cardiac Ang II: $126 \pm 11$ vs $199 \pm 14 \mathrm{pg} / 100 \mathrm{mg}$; cardiac Aldo: $497 \pm 43$ vs $766 \pm 46 \mathrm{pg} / 100 \mathrm{mg}$, all $\mathrm{p}<0.05)$, and the cardiac AT1R protein was down-regulated and AT2R protein was up-regulated, no significant difference of these indices was found between SHR-Aml and untreated SHR. Significance Prehypertensive treatment with losartan was more effective than amlodipine on delaying long-term blood pressure rise and meliorating cardiac structure and function, which might be related to permanent attenuation of circulating and local renin-angiotensin (R-A) systems.

Funding This study was supported by a grant from Education Department of Fujian Province, China (JXK200702).

Competing interests None.

\section{e0161 ESTABLISHMENT OF MINIPIG MODEL OF ISCHAEMIC HEART FAILURE WITH ACUTE MYOCARDIAL INFARCTION BY CORONARY OCCLUSION WITH BALLOON OCCLUDING AND INTERMIXTURE INJECTING OF MICROTHROMBI AND PLASTIC MICROSPHERES}

doi:10.1136/hrt.2010.208967.161

${ }^{1}$ Jing Zhang, ${ }^{2}$ Xianghua Fu, ${ }^{3}$ Oingsheng Wang, ${ }^{3}$ Xinna Fan, ${ }^{2}$ Xuechao Wang, ${ }^{2}$ Yanbo Wang. ${ }^{1}$ The No.1 Hospital of Qinhuangdao; ${ }^{2}$ The 2nd Hospital of Hebei Medical University; ${ }^{3}$ The No1 Hospital of Oinhuangdao

Objective To evaluate the of method of minipig model of ischaemic heart failure (HF) with acute myocardial infarction (AMI) by coronary occlusion with balloon occluding and coadministration injecting of microthrombi and plastic microspheres.

Methods A total of 18 minipigs were selected. After coronary angiography, angioplasty balloons were placed in the mid-distal of left anterior descending (LAD). The balloon was inflated intermittently to occlude the LAD 3 times and then occlude contineously for $120 \mathrm{~min}$. After the balloon was taken out, 4F judkins angiogrphic catheter was superelectively engaged in $\mathrm{LAD}$ and $3 \mathrm{ml}$ intermixture of microthrombi and plastic microspheres were injected at $10 \mathrm{~min}$ interval until TIMI myocardial perfusion grade $<2$ and left ventricular end-diastolic pressure maintaining from 15 to $18 \mathrm{~mm} \mathrm{Hg}$. Electrocardiography (ECG), haemodynamic perameters, ultrasonic cardiogram, cTnI and CK-MB were measured. Myocardiol infarcted area was evaluated with pathologic examination.

Results 14 days later, 15 minipigs survived and fourteen satisfied the criteria (pulmonary capillary wedge pressure, PCWP $>18 \mathrm{~mm} \mathrm{Hg}$ and cardio output, $\mathrm{CO}$ decreased beyond $30 \%$ ). The change of ECG, haemodynamic perameters, CKMB, cTnI and cardiac pathologic examination were in accordance with AMI. Occlusion A stable experimental method of minipig model of ischaemic heart failure (HF) with acute myocardial infarction (AMI) by coronary occlusion with balloon occluding and coadministration injecting of microthrombi and plastic microspheres was established. The method had advantages of closed chest, higher succeed rate and stablility to those of drug induced, tachycardia-pacing induced, coronary artery ligation induced or microsphere injection alone.

\section{e0162 THE RELATIONSHIP BETWEEN THE G (+252)A POLYMORPHISM OF $\beta 2$-ADRENERGIC RECEPTOR GENE AND ESSENTIAL HYPERTENSION IN KAZAKS OF XINJIANG}

doi:10.1136/hrt.2010.208967.162

Liu Kejian, Li Nanfang, Zhou Ling, Yao Xiaoguang, Li Tao, Yan Zhitao. Department of Echocardiography, Center of the Medical Ultrasound, The First Affiliated Hospital of Xinjiang Medical University, Urmuqi, China

Introduction Hypertension is a major risk factor for coronary artery disease, stroke, and renal disease. Recent research suggest genetic polymorphisms of $\beta 2$-adrenergic receptor gene (ADRB2) have already been associated with obesity, diabetes mellitus, and essential 
hypertension, with conflicting results. Kazakans of the pasturing area in Xinjiang, China, have higher prevalence of hypertension and mean blood pressure than other populations of Xinjiang. But up to now no study in this population. To investigate the relationship between the $G(+252)$ A polymarphism of ADRB2 gene and essential hypertension in Kazaks of Xinjiang.

Method A total of 276 patients with confirmed hypertensives and 157 healthy control were genotyped for the G (+252)A by PCR restriction fragment length polymorphism (PCR-RFLP) analysis.

Result Compared with control group, there was no significant difference in the distribution of genotypes and allele frequency of $G$ $(+252)$ A polymorphisms in $\mathrm{EH}$ group $(\mathrm{p}>0.05)$. In addition, age, body mass index, systolic blood pressure and diastolic blood pressure had no significant difference in the groups classified according to genotypes $(\mathrm{p}>0.05)$.

Conclusion G (+252)A polymorphism of $\beta 2$-AR gene was not related with essential hypertension in Kazaks of XinJiang.

\section{e0163 THE ASSOCIATION OF FURIN GENE CODING POLYMORPHISM WITH ESSENTIAL HYPERTENSION IN KAZAKAN OF XINJIANG}

doi:10.1136/hrt.2010.208967.163

Li Nanfang, Luo Wenli, Yao Xiaoguang, Yan Zhitao, Zhang Juhong. Department of Echocardiography, Center of the Medical Ultrasound, The First Affiliated Hospital of Xinjiang Medical University, Urmuqi, China

Introduction The proprotein processing enzyme furin is involved in many blood pressure-regulating factors. In the Golgi, furin appears to activate $\mathrm{ENaC}$. Thus the gene of furin (FUR) may be a candidate gene of hypertension. We investigate the relationship between Furin gene polymorphism and hypertension in Xinjiang Kazakans.

Methods 924 Kazakans was selected randomly from the pasturing area aged in Xinjiang (422 hypertensives, 502 normotensive controls). We sequenced the coding regions of FUR in 94 hypertensive individuals to identify genetic variations of FUR. Genotyping by the TaqMan-PCR method was performed for common SNPs. The possible relationship between the polymorphism and hypertension of Kazakan was analysed.

Results 1 . Direct sequencing from 94 hypertension subjects identified 6 SNPs in the Furin gene promoter. 2. The genotypes and allele frequencies of $-229 \mathrm{C} \rightarrow \mathrm{T}, 12690 \mathrm{G} \rightarrow \mathrm{C}$ polymorphisms are not significant between these two groups. 3. There was no significance of blood pressure among the three genotypes of $-229 \mathrm{C} \rightarrow \mathrm{T}$, $12690 \mathrm{G} \rightarrow \mathrm{C}$ polymorphism. In multiple logistic analyses, the genotypes of the $-229 \mathrm{C}>\mathrm{T}$ were excluded as independent variables. None of haplotypes composed of $-229 \mathrm{C} \rightarrow \mathrm{T}$ and $12690 \mathrm{G} \rightarrow \mathrm{C}$ was significantly different in $\mathrm{EH}$ and controls.

Conclusion The Furin gene $-229 \mathrm{C} \rightarrow \mathrm{T}, 12690 \mathrm{G} \rightarrow \mathrm{C}$ polymorphism might not be associated with essential hypertension in Kazakans.

\section{e0164 DYSREGULATION OF MIR-1 AFTER LEFT VENTRICULAR HYPERTROPHY REVEALS A ROLE OF MIR-1 IN VENTRICULAR ARRHYTHMIA OCCURRED IN THE HYPERTENSIVE HEART}

doi:10.1136/hrt.2010.208967.164

Ying Huang, Weifeng Wu. Department of Cardiology, The First Affiliated Hospital of Guangxi Medical University, Nanning, China

Clinical studies have shown that the rate of VA in hypertensive patients with LVH was several times higher than the normaltensive ones. Currently the mechanism of VA due to LVH is not yet fully clarified. Growing evidences indicate that microRNAs (miRNAs or miRs) are regulators of gene expression, which are becoming increasingly recognised as important regulators of heart function and diseases. Here we observed that miR-1 was striking because of its more than $(2.08 \pm 0.21)$ fold increased in the spontaneously hypertensive rat model (SHRs) with LVH. miR-1 overexpression slowed conduction and depolarised the cytoplasmic membrane by post-transcriptionally repressing Kir2.1 and connexin 43(Cx43), and this likely accounts at least in part for its arrhythmogenic potential. Then we confirmed that in vivo suppression of miR-1 in SHRs could upgrade $\mathrm{Cx} 43$ and Kir2.1 protein level. Our data show that miR-1 is a key regulator of cardiac hypertrophy formation and VA due to $\mathrm{LVH}$, suggesting its attractive therapeutic application in ventricular arrhythmia occurred in the hypertensive heart.

\section{e0165 GENETIC VARIATION OF NEDD4L IS ASSOCIATED WITH ESSENTIAL HYPERTENSION IN FEMALE KAZAKH GENERAL POPULATION}

doi:10.1136/hrt.2010.208967.165

Li Nanfang, Wang Hongmei, Hong Jing, Yao Xiaoguang. Hypertension Unit of the People's Hospital of Xinjiang Uygur Autonomous Region, The Institute of Hypertension of Xinjiang, The Center of Hypertension of the People's Hospital of Xinjiang Uygur Autonomous Region, Urumqi, China

Introduction NEDD4L is a candidate gene for hypertension on both functional and genetic grounds. The study is to investigate the relationships between the variation of NEDD4L and essential hypertension (EH) in Kazakh in China.

Methods We screened the promoter and exons of NEDD4L in 94 Kazakh hypertensive individuals to identify representative variations. Then the representative variations were genotyped in a Kazakh general population, a case-control study was conducted.

Results We did not identify any functional mutations in functional regions of NEDD4L. Three representative variations (296921296923delTTG, rs2288774, rs2288775) were successfully genotyped in Kazakh population. The distribution of the dominant model (AA vs $A G+G G)$ of rs2288775, the additive model and recessive model (II+ID vs DD) of 296921-296923delTTG differed significantly between case and control in female $(p=0.040, p=0.024$ and $p=0.007$ respectively). Logistic regression analysis showed that rs2288775 and 296921-296923delTTG were significantly associated with hypertension ( $\mathrm{rs} 2288775$ : OR $=1.479,95 \%$ CI 1.011 to 2.064 , $p=0.044 ; 296921-296923$ delTTG: $O R=1.908,95 \%$ CI 1.020 to 3.568 , $\mathrm{p}=0.043$ ) in female. The frequency of D-C-G haplotype was significantly higher for case than for control in female $(p=0.020)$.

Conclusions The genetic variations of NEDD4L may be associated with EH in female Kazakh.

\section{e0166 VARIATION OF ADRB2 IS ASSOCIATED WITH COMMON RISK FACTORS FOR CARDIOVASCULAR}

doi:10.1136/hrt.2010.208967.166

Wang Hongmei, Li Nanfang, Hu Junli, Liu Hui. Hypertension Unit of The People's Hospital of Xinjiang Uygur Autonomous Region, The Institute of Hypertension of Xinjiang, The Center of Hypertension of The People's Hospital of Xinjiang Uygur Autonomous Region, Urumqi, China

Introduction Hypertension, overweight/obesity and dyslipidemia are common risk factors for cardiovascular disease. $\beta 2$-adrenergic receptor (ADRB2) regulates blood pressure, lipid mobilisation, and energy expenditure.

Methods A cross-sectional study was conducted in Kazakh of Xinjiang (169 males, 238 females) aged 30 to 60 years. The widely studied polymorphisms A46G, C79G, C491T and A523C in ADRB2 gene were selected to be genotyped by PCR-RFLP. 